\title{
Parity Violation in Elastic Electron-Proton Scattering and the Proton's Strange Magnetic Form Factor
}

\author{
D. T. Spayde, ${ }^{3}$ T. Averett, ${ }^{*}, 1$ D. Barkhuff, ${ }^{4}$ D. H. Beck, ${ }^{2}$ E. J. Beise,${ }^{3}$ C. Benson,${ }^{2}$ H. Breuer, ${ }^{3}$ R. Carr, ${ }^{1}$ S. Covrig, ${ }^{1}$ \\ J. DelCorso, ${ }^{5}$ G. Dodson, ${ }^{4}$ K. Dow, ${ }^{4}$ C. Eppstein, ${ }^{1}$ M. Farkhondeh, ${ }^{4}$ B. W. Filippone, ${ }^{1}$ P. Frazier, ${ }^{1}$ R. Hasty, ${ }^{2}$ T. M. Ito, ${ }^{1}$ \\ C. E. Jones, ${ }^{1}$ W. Korsch, ${ }^{6}$ S. Kowalski, ${ }^{4}$ P. Lee, ${ }^{1}$ E. Maneva, ${ }^{1}$ K. McCarty, ${ }^{1}$ R. D. McKeown, ${ }^{1}$ J. Mikell, ${ }^{2}$ B. Mueller, ${ }^{7}$ \\ P. Naik, ${ }^{2}$ M. Pitt, ${ }^{5}$ J. Ritter, ${ }^{2}$ V. Savu, ${ }^{1}$ M. Sullivan, ${ }^{1}$ R. Tieulent, ${ }^{3}$ E. Tsentalovich,${ }^{4}$ S. P. Wells, ${ }^{8}$ B. Yang, ${ }^{4}$ and T. Zwart ${ }^{4}$ \\ ${ }^{1}$ Kellogg Radiation Laboratory, California Institute of Technology, Pasadena, California 91125 \\ ${ }^{2}$ Department of Physics, University of Illinois at Urbana-Champaign, Urbana, Illinois 61801 \\ ${ }^{3}$ Department of Physics, University of Maryland, College Park, Maryland 20742 \\ ${ }^{4}$ Bates Linear Accelerator Center, Laboratory for Nuclear Science and Department of Physics, \\ Massachusetts Institute of Technology, Cambridge, Massachusetts 02139 \\ ${ }^{5}$ Department of Physics, Virginia Polytechnic Institute and State University, Blacksburg, Virginia 24061-0435 \\ ${ }^{6}$ Department of Physics and Astronomy, University of Kentucky, Lexington, Kentucky 40506 \\ ${ }^{7}$ Physics Division, Argonne National Laboratory, Argonne, Illinois 60439 \\ ${ }^{8}$ Department of Physics, Louisiana Tech University, Ruston, Louisiana 71272
}

(SAMPLE Collaboration)

(Received 15 September 1999)

\begin{abstract}
We report a new measurement of the parity-violating asymmetry in elastic electron scattering from the proton at backward scattering angles. This asymmetry is sensitive to the strange magnetic form factor of the proton as well as electroweak axial radiative corrections. The new measurement of $A=$ $-4.92 \pm 0.61 \pm 0.73 \mathrm{ppm}$ provides a significant constraint on these quantities. The implications for the strange magnetic form factor are discussed in the context of theoretical estimates for the axial corrections.
\end{abstract}

PACS numbers: 13.60.Fz, 11.30.Er, 13.40.Gp, 14.20.Dh

The anomalous magnetic moments of the neutron and proton are important clues to their internal quark structure. Since the first measurement of the proton's magnetic moment in 1933, our empirical knowledge of the electromagnetic structure of the nucleon has been greatly improved through detailed measurements of the electric and magnetic form factors and their four-momentum transfer $\left(Q^{2}\right)$ dependence. Nevertheless, we still lack a quantitative theoretical understanding of these properties (including the magnetic moments), and additional experimental information is crucial in our effort to understand the internal structure of the nucleon.

Whereas the normal magnetic moment corresponds to the magnetic coupling to the photon, the weak magnetic moment represents the analogous coupling to the $Z$ boson, equally fundamental and just as important as the electromagnetic moment. The weak magnetic form factor provides unambiguous new information about the quark flavor structure of the nucleon and enables a complete decomposition of its magnetic structure into the contributions from different quark flavors (up, down, and strange) [1]. To lowest order, the neutral weak magnetic form factor of the proton, $G_{M}^{Z}$, is related to nucleon electromagnetic form factors and a contribution from strange quarks:

$$
G_{M}^{Z}=\left(G_{M}^{p}-G_{M}^{n}\right)-4 \sin ^{2} \theta_{W} G_{M}^{p}-G_{M}^{s},
$$

where $G_{M}^{p}$ and $G_{M}^{n}$ are the (electromagnetic) magnetic form factors of the proton and neutron, $\theta_{W}$ is the weak mix- ing angle, and $G_{M}^{s}$ is the contribution from strange quarks. (Electroweak radiative corrections to this expression have been computed in Ref. [2].) Thus the measurement of $G_{M}^{Z}$ provides unique access to the strange quark-antiquark "sea" and its role in the basic electromagnetic structure of the nucleon at low energies. $G_{M}^{Z}$ can be determined via parity-violating elastic electron-proton scattering [3]. (Note: This definition of $G_{M}^{Z}$ differs by a factor of 4 from that used in Ref. [4] in order to conform with more standard notation in the literature. The definition of $G_{M}^{s}$ is the same as in Ref. [4].)

In this Letter, we report a new measurement of the parity-violating asymmetry with sufficient precision to provide significant quantitative information on $G_{M}^{s}$. In comparison to our previous results [4], this measurement has involved both improved monitoring and control of systematic errors as well as better statistical precision.

As previously discussed [3], the parity-violating asymmetry for elastic scattering of right- vs left-handed electrons from nucleons at backward scattering angles is sensitive to $G_{M}^{Z}$. The SAMPLE experiment measured the parity-violating asymmetry in the elastic scattering of $200 \mathrm{MeV}$ longitudinally polarized electrons from protons at backward angles with an average $Q^{2} \simeq 0.1(\mathrm{GeV} / c)^{2}$. For $G_{M}^{s}=0$, the expected asymmetry in the SAMPLE experiment is about $-7 \times 10^{-6}$ or $-7 \mathrm{ppm}$, and the asymmetry depends linearly on $G_{M}^{s}$. The neutral weak axial form factor $G_{A}^{Z}$ contributes about $20 \%$ to the asymmetry 
in our experiment. In parity-violating electron scattering $G_{A}^{Z}$ is modified by a substantial electroweak radiative correction. The corrections were estimated in [2], but there is considerable uncertainty in the calculations. The uncertainty in these radiative corrections substantially limits our ability to determine $G_{M}^{s}$, as will be discussed below.

The SAMPLE experiment was performed at the MIT/ Bates Linear Accelerator Center using a $200 \mathrm{MeV}$ polarized electron beam incident on a liquid hydrogen target. The scattered electrons were detected in a large solid angle $(\sim 1.5 \mathrm{sr})$ air Čerenkov detector. The detector consists of ten large mirrors, each with ellipsoidal curvature to focus the Čerenkov light onto one of ten shielded photomultiplier tubes. A remotely controlled light shutter can cover each photomultiplier tube for background measurements. Typically one-fourth of the data was taken with shutters closed to monitor this background. As described in Ref. [4], the Čerenkov detector signals were studied at low beam intensity to determine the composition of the signal, including the fraction of light due to elastic scattering (factors that scaled the individual mirror asymmetries by typically 1.8 , depending upon the mirror, and that were determined to a precision of $4 \%$ ). The parity-violating asymmetry was measured using higher beam currents, for which it was necessary to integrate the detector signals over the beam pulse. The incident electron beam ( $2.7 \mathrm{~mA}$ peak current) was pulsed at $600 \mathrm{~Hz}$; the signals from the detector, beam toroid monitors, and various other beam monitors were integrated and digitized for every $25 \mu \mathrm{sec}$ long beam pulse. The parity-violating asymmetry $A$ was determined from the asymmetries in ratios of integrated detector signal to beam intensity for left- and right-handed beam pulses.

The polarized electron beam was generated via photoemission from unstrained GaAs by polarized laser light. The laser beam helicity for each pulse was determined by a $\lambda / 4$ Pockels cell and was randomly chosen for each of 10 consecutive beam pulses; the complement helicities were then used for the next 10 pulses. The asymmetry in the normalized detector yields was computed for "pulse pairs" separated by $1 / 60$ of a second to minimize systematic errors. The electron beam helicity relative to all electronic signals can be manually reversed by inserting a $\lambda / 2$ plate in the laser beam. (We denote this configuration as $\lambda / 2=$ "IN" as opposed to $\lambda / 2=$ "OUT".) During the 1998 running period, the IN/OUT configuration was reversed every few days to minimize false asymmetries and test for systematic errors. The electron polarization was measured using a Møller system on the beam line and averaged $36.3 \pm 1.4 \%$ during the experiment. The effect of small transverse components of electron polarization on the observed parity violation signal was studied and determined to be negligible.

Helicity correlations of various parameters of the electron beam were monitored continuously during the experiment. These parameters include the beam intensity, position, and angle at the target in both transverse dimen- sions ( $x$ and $y$ ), the beam energy, and the beam "halo." Two forward angle lucite Cerenkov counters were also implemented at $\sim 12^{\circ}$ to monitor luminosity and test for helicity dependence. These monitors detected low $Q^{2}$ elastic scattering at forward angles and other soft electromagnetic radiation and should show negligible parity violating asymmetry.

As in the past, we reduced the beam intensity asymmetry through an active feedback system. In 1998, this feedback was implemented with an additional Pockels cell located between linear polarizers to separate this function from the Pockels cell that controlled the helicity (HPC). The HPC was also repositioned to be downstream of all laser transport elements. These changes resulted in improved stability of the laser beam position under helicity reversal. In addition, we implemented a feedback system to reduce the remaining helicity-correlated beam position asymmetry [5]. This was accomplished using a tilted glass plate in the laser beam path and a piezoelectric transducer. By adjusting the tilt of this glass plate with helicity reversal, the first-order beam position asymmetry is reduced, resulting in improved quality of the data. For example, the helicity correlated vertical beam shift at the target was reduced from $\sim 200 \mathrm{~nm}$ to typically $<20 \mathrm{~nm}$.

Of the 110 coulombs (C) of beam delivered to the experiment in 1998, the first $24 \mathrm{C}$ were taken before the position feedback system was fully implemented, and significant position asymmetries were present. This is evident in Fig. 1a, where the luminosity monitor asymmetries are shown for this time frame ("piezo off") in comparison with the later runs ("piezo on"). A linear regression technique is used to remove such effects from the data [6]. The results of this analysis are shown in Fig. 1b, where the corrected asymmetries are displayed. This procedure (involving six beam parameters: $x, y, \theta_{x}$, $\theta_{y}$, energy, and intensity) is very effective at removing the effects of beam helicity correlations, resulting in a final corrected luminosity monitor asymmetry result of $0.17 \pm 0.11 \mathrm{ppm}$. In Fig. 2 are shown the analogous plots for the asymmetry measured in the SAMPLE detector (all ten mirrors combined and corrected for background dilution, radiative effects, and beam polarization). In contrast to the luminosity monitors, the detector asymmetry is quite robust with respect to beam helicity correlations, the corrections affecting the final asymmetry by only $5 \%$, or $0.2 \mathrm{ppm}$. This correction is about equal to the estimated systematic error in the procedure as determined from the luminosity monitor analysis.

The elastic scattering asymmetry was determined from the 10 individual mirror asymmetries after correction for all effects, including background dilution. The measured shutter closed asymmetry for all 10 mirrors combined (appropriately scaled to compare directly to the elastic asymmetry), is $-0.57 \pm 0.64 \mathrm{ppm}$, consistent with zero as expected, assuming the shutter closed yield is dominated by low- $Q^{2}$ processes. However, the mirror-by-mirror 

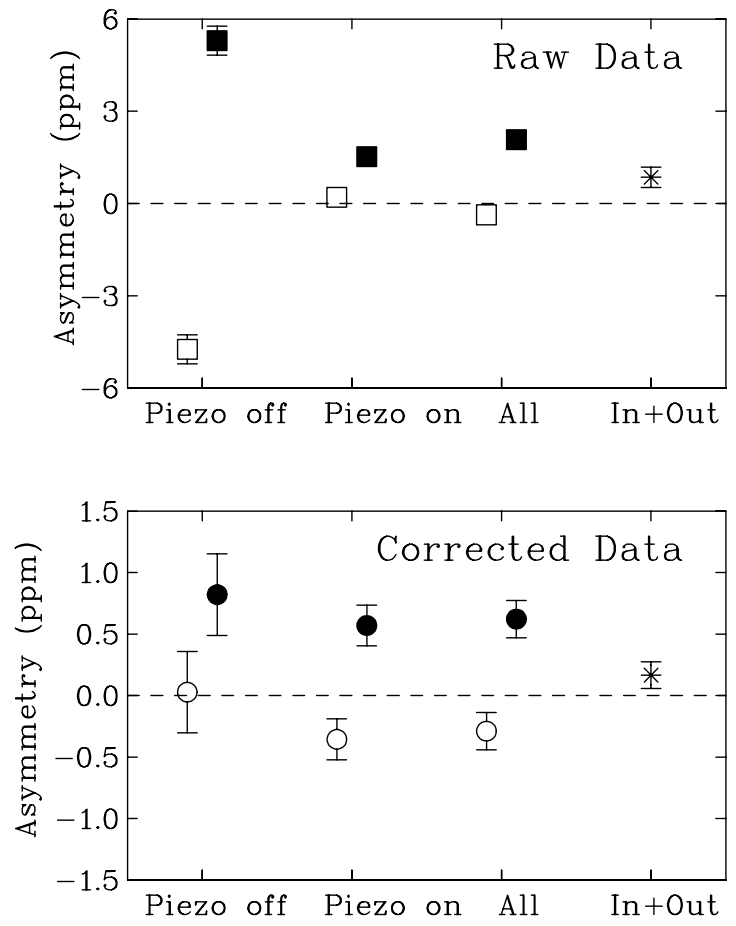

FIG. 1. Measured asymmetries in the luminosity monitors in parts per million (ppm). The open symbols are for $\lambda / 2=$ IN and the filled symbols are for $\lambda / 2=$ OUT. The upper plot shows the raw data and the lower plot shows the data corrected for beam parameter asymmetries. The beam polarization is accounted for and the error bars include statistical errors only. Note the change in scale in the lower plot.

distribution of shutter closed asymmetries is statistically improbable, indicating the presence of some nonstatistical component to the shutter closed yield. We therefore assume the combined shutter closed asymmetry to be zero in our analysis, and assign a systematic error due to the uncertainty in the shutter closed asymmetry of $0.64 \mathrm{ppm}$.

The resulting elastic asymmetry is

$$
A=-4.92 \pm 0.61 \pm 0.73 \mathrm{ppm},
$$

where the first uncertainty is statistical and the second is the estimated systematic error as summarized in Table I. This value is in good agreement with our previous reported measurement [4].

At the mean kinematics of the experiment $\left[Q^{2}=\right.$ $0.1(\mathrm{GeV} / c)^{2}$ and $\left.\theta=146.1^{\circ}\right]$, the theoretical asymmetry is

$$
A=-5.61+3.49 G_{M}^{s}+1.55 G_{A}^{Z},
$$

where

$$
G_{A}^{Z}=-\left(1+R_{A}^{1}\right) G_{A}+R_{A}^{0}+G_{A}^{s} .
$$

We estimate the error in the constant term of $A$, dominated by uncertainties in the electromagnetic form factors, to be 3\%. $G_{A}$ is the charged current nucleon form factor: we use $G_{A}=G_{A}(0) /\left(1+\frac{Q^{2}}{M_{A}^{2}}\right)^{2}$, with $G_{A}(0)=-\left(g_{A} / g_{V}\right)=$
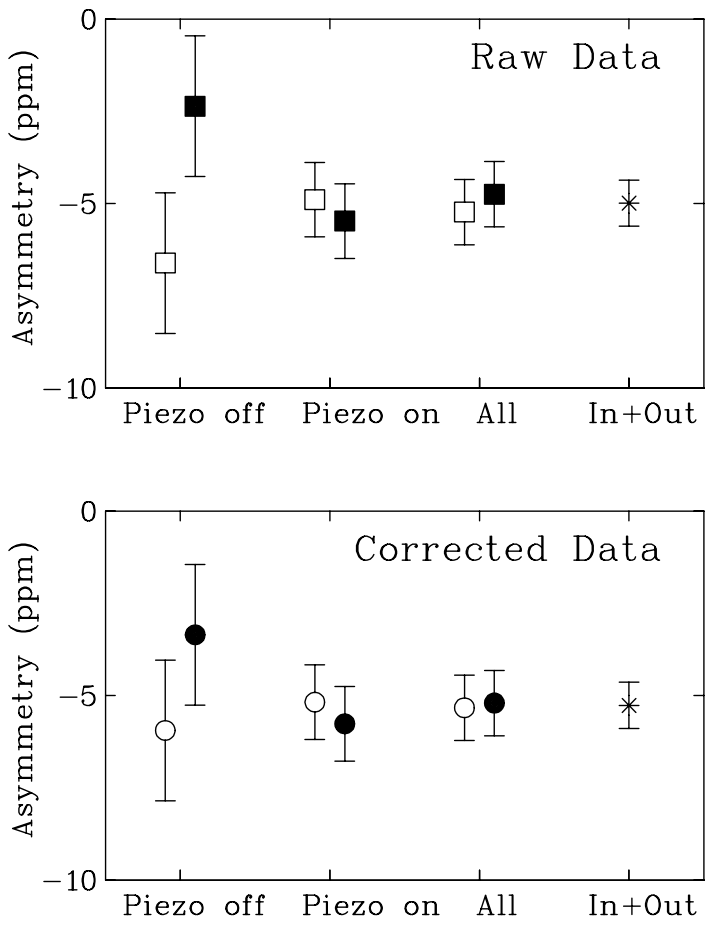

FIG. 2. Measured asymmetries for the combined Čerenkov detectors. The open symbols are for $\lambda / 2=\mathrm{IN}$ and the filled symbols are for $\lambda / 2=$ OUT. The upper plot shows the raw data and the lower plot shows the data corrected for beam parameter asymmetries. The error bars are statistical only. Including (mirror-by-mirror) the systematic error changes the relative weights of each mirror, resulting in the slightly smaller value of $A$ in Eq. (2).

$1.2670 \pm 0.0035[7]$ and $M_{A}=1.069 \pm 0.016(\mathrm{GeV} / c)$ [8]. The strange axial form factor $G_{A}^{s}$ is taken to be the value of $\Delta s=-0.1$ extracted from polarized deepinelastic lepton scattering. Because extraction of $\Delta s$ depends on assumptions about $\mathrm{SU}(3)$ flavor symmetry, and because of the unknown $Q^{2}$ dependence of $G_{A}^{s}$, we assign a $100 \%$ error on this value [9]. The isoscalar and isovector axial radiative corrections $R_{A}^{0,1}$ were estimated by Ref. [2] to be $R_{A}^{1}=-0.34 \pm 0.28$ and $R_{A}^{0}=-0.12 \pm$ 0.12 . [The notation used here is $R_{A}^{0}=\sqrt{3} G_{A}^{8} R_{A}^{T=0}$, where $G_{A}^{8}$ is the $\mathrm{SU}(3)$ isoscalar octet form factor estimated from hyperon beta decay and $\sqrt{3} R_{A}^{T=0}=-0.62$ as discussed in Ref. [2(b)].]

TABLE I. Summary of relative uncertainties on the measured asymmetry.

\begin{tabular}{lc}
\hline \hline \multicolumn{1}{c}{ Source } & $\delta A / A(\%)$ \\
\hline Shutter closed asymmetries & 13 \\
Helicity-correlated corrections procedure & 5 \\
Unpolarized background dilution factor & 4 \\
Beam polarization determination & 4 \\
Total systematic (added in quadrature) & 15 \\
\hline \hline
\end{tabular}




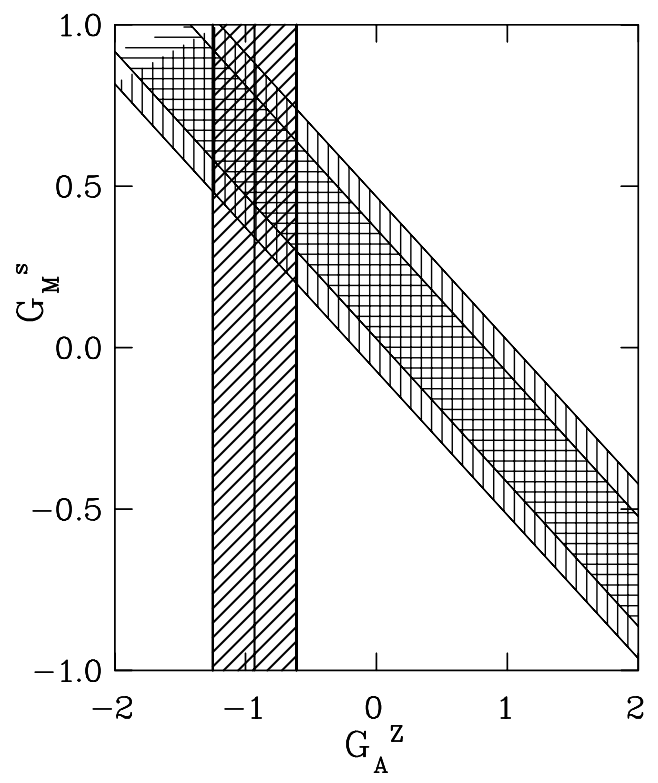

FIG. 3. Error band of $G_{M}^{s}$ for the allowed region (shaded) corresponding to the present measurement of the parity-violating asymmetry. The inner hatched region includes the statistical error, the outer represents the systematic uncertainty added in quadrature, and the vertical band corresponds to the calculated value of $G_{A}^{Z}$ [as defined in Eq. (4)] using theoretical estimates of Ref. [2] for $R_{A}^{0}$ and $R_{A}^{1}$ with their estimated errors.

The strange magnetic form factor derived from the asymmetry in Eq. (2) is

$$
\begin{aligned}
G_{M}^{s}\left[Q^{2}=0.1(\mathrm{GeV} / c)^{2}\right]= & -0.45 G_{A}^{Z}+0.20 \\
& \pm 0.17 \pm 0.21 .
\end{aligned}
$$

This result is graphically displayed in Fig. 3, along with $G_{A}^{Z}$ (vertical band) computed from taking the above values for $\Delta s, R_{A}^{0}$, and $R_{A}^{1}$, with their errors added quadratically. Combining this band for $G_{A}^{Z}$ with our measurement implies a substantially positive value of $G_{M}^{s}\left[Q^{2}=\right.$ $\left.0.1(\mathrm{GeV} / c)^{2}\right]=+0.61 \pm 0.17 \pm 0.21 \pm 0.19$, where the last error is due to the inferred uncertainty in $G_{A}^{Z}$. As noted in recent papers [10,11], most model calculations tend to produce negative values of $\mu_{s} \equiv G_{M}^{s}\left(Q^{2}=0\right)$, typically about -0.3 . A recent calculation using lattice QCD techniques (in the quenched approximation) reports a result $\mu_{s}=-0.36 \pm 0.20$ [11]. As shown in Fig. 3, our new measurement implies that the computed negative value of $G_{A}^{Z}$ is inconsistent with $G_{M}^{s}<0$. Another recent study using a constrained Skyrme-model Hamiltonian that fits the baryon magnetic moments yields a positive value of $\mu_{s}=+0.37$ [12], which is in better agreement with our measurement and with the calculated value of $G_{A}^{Z}$.

Since the dominant uncertainty in $G_{A}^{Z}$ comes from $R_{A}^{1}$, eliminating the uncertainty in $R_{A}^{1}$ is essential for deriving a firm conclusion about $G_{M}^{s}$. Toward this end, we are presently running the SAMPLE experiment with a deuterium target to measure the quasielastic asymmetry from deuterium [13]. This asymmetry is quite insensitive to strange quark effects and will therefore independently determine the isovector axial radiative correction. When the deuterium data are available, we will be able to provide definitive experimental information on the proton's strange magnetic form factor.

The skillful efforts of the staff of the MIT/Bates facility to provide high quality beam and improve the experiment are gratefully acknowledged. This work was supported by NSF Grants No. PHY-9420470 (Caltech), No. PHY-9420787 (Illinois), No. PHY-9457906/PHY9971819 (Maryland), No. PHY-9733772 (VPI), and DOE Cooperative Agreement No. DE-FC02-94ER40818 (MITBates) and Contract No. W-31-109-ENG-38 (ANL).

*Present address: Department of Physics, College of William and Mary, Williamsburg, VA 23187.

[1] D. Kaplan and A. Manohar, Nucl. Phys. B310, 527 (1988).

[2] (a) M. J. Musolf and B. R. Holstein, Phys. Lett. B 242, 461 (1990); (b) M. J. Musolf et al., Phys. Rep. 239, 1 (1994).

[3] R. D. McKeown, Phys. Lett. B 219, 140 (1989); D. H Beck, Phys. Rev. D 39, 3248 (1989).

[4] B. A. Mueller et al., Phys. Rev. Lett. 78, 3824 (1997).

[5] T. Averett et al., Nucl. Instrum. Methods Phys. Res., Sect. A 438, 246 (1999).

[6] B. A. Mueller, Ph.D. thesis, California Institute of Technology, 1997 (unpublished).

[7] Particle Data Group, C. Caso et al., Eur. Phys. J. C3, 1 (1998).

[8] A. Liesenfeld et al., Phys. Lett. B 468, 19 (1999).

[9] See, for example, J. Dai, R. Dashen, E. Jenkins, and A. V. Manohar, Phys. Rev. D 53, 273 (1996); J. Lichtenstadt and H. Lipkin, Phys. Lett. B 353, 119 (1995), and references therein.

[10] R. D. McKeown, in Parity Violation in Atoms and Polarized Electron Scattering, edited by B. Frois and M. A. Bouchiat (World Scientific, Singapore, 1999), p. 423.

[11] S. J. Dong, K. F. Liu, and A. G. Williams, Phys. Rev. D 58, 074504 (1998).

[12] S-T. Hong, B-Y. Park, and D-P. Min, Phys. Lett. B 414, 229 (1997).

[13] Bates experiment 94-11 (M. Pitt and E. J. Beise, spokespersons). 